OSU-HEP-05-11

LPT-ORSAY-05-62

\title{
Leptogenesis in Minimal Left-Right Symmetric Models
}

\author{
K.S. Babu ${ }^{1}$ and A. Bachri ${ }^{2}$ \\ Oklahoma Center for High Energy Physics \\ Department of Physics, Oklahoma State University \\ Stillwater, OK 74078, USA \\ H. Aissaoui ${ }^{3}$ \\ Laboratoire de Physique Théorique, Université de Paris XI, \\ Bâtiment 210, 91405 Orsay Cedex, France \\ Laboratoire de Physique Mathématique et Physique Subatomique \\ Mentouri University, Constantine 25000 Algeria
}

\begin{abstract}
We analyze lepton asymmetry induced in the decay of right-handed neutrinos in a class of minimal left-right symmetric models. In these models, which assume low energy supersymmetry, the Dirac neutrino mass matrix is proportional to the charged lepton mass matrix. As a result, lepton asymmetry is calculable in terms of 9 parameters, all measurable in low energy neutrino experiments. By solving the Boltzmann equations numerically we show that adequate baryon asymmetry is generated in these models. This however places significant constraints on the light neutrino parameters. We find $\tan ^{2} \theta_{12} \simeq m_{1} / m_{2}$ and $\theta_{13}=(0.01-0.07)$ for the neutrino oscillation angles, and $\beta \simeq \alpha+\pi / 2$ for the Majorana phases.
\end{abstract}

\footnotetext{
${ }^{1}$ kaladi.babu@okstate.edu

2 abdelghafour.bachri@okstate.edu

3 aissaoui@th.u-psud.fr
} 


\section{Introduction}

The discovery of neutrino flavor oscillations in solar, atmospheric, and reactor neutrino experiments 1] may have a profound impact on our understanding of the dynamics of the early universe. This is because such oscillations are feasible only if the neutrinos have small (sub-eV) masses, most naturally explained by the seesaw mechanism [2]. This assumes the existence of super-heavy right-handed neutrinos $N_{i}$ (one per lepton family) with masses of order $\left(10^{8}-10^{14}\right) \mathrm{GeV}$. The light neutrino masses are obtained from the matrix $M_{\nu} \simeq M_{D} M_{R}^{-1} M_{D}^{T}$ where $M_{D}$ and $M_{R}$ are respectively the Dirac and the heavy Majorana right-handed neutrino (r.h.n) mass matrices. The decay of the lightest right-handed neutrino $N_{1}$ can generate naturally an excess of baryons over anti-baryons in the universe [3] consistent with cosmological observations. The baryon asymmetry parameter is an important cosmological observable constrained by Big Bang Nucleosynthesis and determined recently with high precision by the WMAP experiment [4]:

$$
\eta_{B} \equiv \frac{n_{B}}{n_{\gamma}}=\left(6.5_{-0.3}^{+0.4}\right) \times 10^{-10}
$$

The decay of $N_{1}$ can satisfy all three of the Sakharov conditions [5] needed for successful generation of $\eta_{B}$ - it can occur out of thermal equilibrium, there is sufficient $C$ and $C P$ violation, and there is also baryon number violation. The last condition is met by combining lepton number violation in the Majorana masses of the right-handed neutrinos with $B+$ $L$ violating interactions of the Standard Model arising through the electroweak sphaleron processes [6]. A compelling picture emerges, with the same mechanism explaining the small neutrino masses and the observed baryon asymmetry of the universe. $\eta_{B}$ appears to be intimately connected to the observed neutrino masses and mixings.

A more careful examination of the seesaw structure would reveal that, although there is an underlying connection, the light neutrino mass and mixing parameters cannot determine the cosmological baryon asymmetry, when the seesaw mechanism is implemented in the context of the Standard Model (SM) gauge symmetry. It is easy to see this as follows. Without loss of generality one can work in a basis where the charged lepton mass matrix and the heavy right-handed neutrino Majorana mass matrix $M_{R}$ are diagonal with real eigenvalues. 
The Dirac neutrino mass matrix would then be an arbitrary complex $3 \times 3$ matrix with 18 parameters (9 magnitudes and 9 phases). Three of the phase parameters can be removed by field redefinitions of the left-handed lepton doublets and the right-handed charged lepton singlets. The neutrino sector will then have $18(=15+3)$ parameters. 9 combinations of these will determine the low energy observables (3 masses, 3 mixing angles and 3 phases), while the lepton asymmetry (and thus $\eta_{B}$ ) would depend on all 18 parameters, leaving it arbitrary.

In this paper we show that it is possible to quantitatively relate $\eta_{B}$ to light neutrino mass and mixing parameters by implementing the seesaw mechanism in the context of a class of supersymmetric left-right models [7]. We note that unlike in the SM where the righthanded neutrinos appear as rather ad hoc additions, in the left-right symmetric models they are more natural as gauge invariance requires their existence. Supersymmetry has the wellknown merit of solving the gauge hierarchy problem. With the assumption of a minimal Higgs sector, it turns out that these models predict the relation for the Dirac neutrino mass matrix

$$
M_{D}=c\left(\begin{array}{ccc}
m_{e} & 0 & 0 \\
0 & m_{\mu} & 0 \\
0 & 0 & m_{\tau}
\end{array}\right)
$$

where $c \simeq m_{t} / m_{b}$ is determined from the quark sector, leaving only the Majorana mass matrix $M_{R}$ to be arbitrary. 3 phases in $M_{R}$ can be removed, leaving a total of 9 parameters which determine both the low energy neutrino masses and mixings as well as the baryon asymmetry. It then becomes apparent that $\eta_{B}$ is calculable in terms of the neutrino observables. There have been other attempts in the literature to relate leptogenesis with low energy observables [8, 9]. Such attempts often make additional assumptions such as $M_{D}=M_{\text {up }}$ (which may not be fully realistic), or specific textures for lepton mass matrices.

While a lot has been learned from experiments about the light neutrino masses and mixings, a lot remains to be learned. Our analysis shows that cosmology puts significant restrictions on the light neutrino parameters. Successful baryogenesis requires within our model that three conditions be satisfied: $\tan ^{2} \theta_{12} \simeq m_{1} / m_{2}, \beta \simeq \alpha+\pi / 2$ and $\theta_{13}=(0.01-$ 0.07). Here $\theta_{12}$ and $\theta_{13}$ are elements of the neutrino mixing matrix, $m_{i}$ are the light neutrino 
mass eigenvalues and $\alpha, \beta$ are the Majorana phases entering in the amplitude for neutrinoless double beta decay. Future neutrino experiments will be able to either confirm or refute these predictions.

The rest of the paper is organized as follows. In Sec. 2 we review briefly the minimal left-right symmetric model. In Sec. 3 we analyze leptogenesis in this model. Here we derive constraints imposed on the model from the requirement of successful leptogenesis. In Sec. 4 we calculate the lepton asymmetry parameter $\varepsilon_{1}$ generated in the model in $N_{1}$ decay. Sec. 5 summarizes the relevant Boltzmann equations needed for computing the baryon asymmetry parameter. Sec. 6 provides our numerical results for $\eta_{B}$. Finally, in Sec. 7 we conclude.

\section{Brief review of the minimal left-right symmetric model}

Let us briefly review the basic structure of the minimal SUSY left-right symmetric model developed in Ref. [7]. The gauge group of the model is $S U(3)_{C} \times S U(2)_{L} \times S U(2)_{R} \times U(1)_{B-L}$. The quarks and leptons are assigned to the gauge group as follows. Left-handed quarks and leptons $(Q, L)$ transform as doublets of $S U(2)_{L}[Q(3,2,1,1 / 3)$ and $L(1,2,1,-1)]$, while the right-handed ones $\left(Q^{c}, L^{c}\right)$ are doublets of $S U(2)_{R}\left[Q^{c}\left(3^{*}, 1,2,-1 / 3\right)\right.$ and $\left.L^{c}(1,1,2,1)\right]$. The Dirac masses of fermions arise through their Yukawa couplings to a Higgs bidoublet $\Phi(1,2,2,0)$. The $S U(2)_{R} \times U(1)_{B-L}$ symmetry is broken to $U(1)_{Y}$ by the $\operatorname{VEV}\left(v_{R}\right)$ of a $B-L=-2$ triplet scalar field $\Delta^{c}(1,1,3,-2)$. This triplet is accompanied by a left-handed triplet $\Delta(1,3,1,2)$ (along with $\bar{\Delta}$ and $\bar{\Delta}^{c}$ fields, their conjugates to cancel anomalies). These fields also couple to the leptons and are responsible for inducing large Majorana masses for the $\nu_{R}$. An alternative to these triplet Higgs fields is to use $B-L= \pm 1$ doublets $\chi(1,2,1,-1)$ and $\chi^{c}(1,1,2,1)$, along with their conjugates $\bar{\chi}$ and $\bar{\chi}^{c}$. In this case non-renormalizable operators will have to be invoked to generate large $\nu_{R}$ Majorana masses. For definiteness we shall adopt the triplet option, although our formalism allows for the addition of any number of doublet Higgs fields as well. The superpotential invariant under the gauge symmetry involving the quark and lepton fields is

$$
W=\mathbf{Y}_{q} Q^{T} \tau_{2} \Phi \tau_{2} Q^{c}+\mathbf{Y}_{l} L^{T} \tau_{2} \Phi \tau_{2} L^{c}+\left(\mathbf{f} L^{T} i \tau_{2} \Delta L+\mathbf{f}_{c} L^{c T} i \tau_{2} \Delta^{c} L^{c}\right)
$$


Under left-right parity symmetry, $Q \leftrightarrow Q^{c *}, L \leftrightarrow L^{c *}, \Phi \leftrightarrow \Phi^{\dagger}, \Delta \leftrightarrow \Delta^{c *}$, along with $W_{S U(2)_{L}} \leftrightarrow W_{S U(2)_{R}}^{*}, W_{B-L} \leftrightarrow W_{B-L}^{*}$ and $\theta \leftrightarrow \bar{\theta}$. As a consequence, $\mathbf{Y}_{q}=\mathbf{Y}_{q}^{\dagger}, \mathbf{Y}_{l}=\mathbf{Y}_{l}^{\dagger}$, and $\mathbf{f}=\mathbf{f}_{c}^{*}$ in Eq. (3) ${ }^{4}$ It has been shown in Ref. [7] that the hermiticity of the Yukawa matrices (along with the parity constraints on the soft SUSY breaking parameters) helps to solve the supersymmetric CP problem that haunts the MSSM.

Below $v_{R}$, the effective theory is the MSSM with its $H_{u}$ and $H_{d}$ Higgs multiplets. ${ }^{5}$ These are contained in the bidoublet $\Phi$ of the SUSY left-right model, but in general they can also reside partially in other multiplets having identical quantum numbers under the MSSM symmetry (such as the $\chi, \bar{\chi}$ doublet Higgs fields alluded to earlier). Allowing for such a possibility, the superpotential of Eq. (3) leads to the relations for the MSSM Yukawa coupling matrices

$$
\mathbf{Y}_{u}=\gamma \mathbf{Y}_{d}, \quad \mathbf{Y}_{\ell}=\gamma \mathbf{Y}_{\nu^{D}}
$$

These relations have been called up-down unification [7. Here, the first relation of Eq. (44) implies $\frac{m_{t}}{m_{b}} \simeq \gamma \tan \beta \equiv c$ where $\gamma$ is a parameter characterizing how much of $H_{u}$ and $H_{d}$ of MSSM are in the bidoublet $\Phi$. The case of $H_{u, d}$ entirely in $\Phi$ will correspond to $\gamma=1$ and $\tan \beta=m_{t} / m_{b}$. At first sight the first of the relations in Eq. (41) might appear phenomenologically disastrous since it leads to vanishing quark mixings and unacceptable quark mass ratios. It was shown in the first paper of Ref. [7] that including the one-loop diagrams involving the gluino and the chargino and allowing for a flavor structure for the soft SUSY breaking $A$ terms, there exists a large range of parameters (though not the entire range possible in the usual MSSM) where correct quark mixings as well as masses can be obtained consistent with flavor changing constraints.

It is the second of Eq. (4) that concerns us here. This relation would lead to $M_{D}=c M_{l}$, with $c \simeq m_{t} / m_{b}$. One can thus go to a basis where the charged lepton and the Dirac neutrino mass matrices are simultaneously diagonal. The heavy Majorana mass matrix $M_{R}=\mathbf{f} v_{R}$ will then be a generic complex symmetric matrix. After removing three phases in $M_{R}$ by field

\footnotetext{
${ }^{4}$ We do not explicitly use these relations.

${ }^{5}$ The right-handed gauge bosons have masses of order $v_{R} \sim 10^{14} \mathrm{GeV}$ and thus play no significant role in cosmology at $T \sim M_{1} \ll v_{R}$.
} 
redefinitions, we are left with 9 parameters (6 magnitudes and 3 phases) which determine the light neutrino spectrum as well as the heavy neutrino spectrum. This in turns fixes the lepton asymmetry. The consequences of such a constrained system for leptogenesis will be analyzed in the next section.

In principle the $\Delta(1,3,1,+2)$ Higgs field can also acquire a small $\mathrm{VEV} \lesssim \mathcal{O}(\mathrm{eV})[11$. In this case the seesaw formula would be modified, as will the calculation of the lepton asymmetry [11. We will assume such type II seesaw contributions proportional to $\langle\Delta\rangle$ are zero in our analysis. This is consistent with the models of Ref. [7. Leptogenesis in the context of more general left-right symmetric models has been analyzed in Ref. [12].

\section{Leptogenesis in left-right symmetric framework}

The $S U(2)_{R} \times U(1)_{B-L}$ symmetry is broken down to $U(1)_{Y}$ by the $\operatorname{VEV}\left\langle\Delta^{c}\right\rangle=v_{R} \sim$ $10^{14} \mathrm{GeV}$. At least some of the right-handed neutrinos have masses below $v_{R}$. We thus focus on the neutrino Yukawa coupling in the context of MSSM. The $S U(2)_{L} \times U(1)_{Y}$ invariant Yukawa interactions are contained in the MSSM superpotential

$$
W=l H_{d} \mathbf{Y}_{\ell} e^{c}+l H_{u} \mathbf{Y}_{\nu^{D}} \nu^{c}+\frac{1}{2} \underbrace{\nu^{c T} C M_{R} \nu^{c}}
$$

where $l$ stands for the left-handed lepton doublet, and $\left(e^{c}, \nu^{c}\right)$ denote the conjugates of the right-handed charged lepton and the right-handed neutrino fields respectively. $H_{u}, H_{d}$ are the MSSM Higgs fields with VEVs $v_{u}, v_{d} . M_{l}=\mathbf{Y}_{\ell} v_{d}, M_{D}=\mathbf{Y}_{\nu^{D}} v_{u}$ and $M_{R}$ are respectively the charged lepton, the Dirac neutrino, and the Majorana r.h.n mass matrices. Then one can generate light neutrino masses by the seesaw mechanism [2]

$$
M_{\nu}=-M_{D} M_{R}^{-1} M_{D}^{T}
$$

There is mixing among generations in both $M_{R}$ and $M_{D}$, the light neutrino mixing angles will depend on both of these mixings. Within the SM or MSSM where $M_{D}$ is an arbitrary matrix, the structure of the right-handed neutrino mass matrix can not be fully determined even if the light matrix $M_{\nu}$ were to be completely known from experiments. As noted in 
Sec. 2 , in the minimal version of the left-right symmetric model one has

$$
M_{D}=c M_{l}=c \operatorname{diag}\left(m_{e}, m_{\mu}, m_{\tau}\right)
$$

where $c \simeq \frac{m_{t}}{m_{b}}$. Here we have already gone to a basis where the charged lepton mass matrix is diagonalized. In the three family scenario, the relations between the flavor eigenstates $\left(\nu_{e}, \nu_{\mu}, \nu_{\tau}\right)$ and the mass eigenstates $\left(\nu_{1}, \nu_{2}, \nu_{3}\right)$ can be expressed in terms of observables as

$$
M_{\nu}=U^{*} M_{\nu}^{\operatorname{diag}} U^{\dagger}
$$

where $M_{\nu}^{\text {diag }} \equiv \operatorname{diag}\left(m_{1}, m_{2}, m_{3}\right)$, with $m_{i}$ being the light neutrinos masses and $U$ being the $3 \times 3$ mixing matrix which we write as $U=U_{P M N S} . P$. We parameterize $U_{P M N S}$ [13] as

$$
\begin{aligned}
U_{P M N S} & =\left(\begin{array}{ccc}
U_{e 1} & U_{e 2} & U_{e 3} \\
U_{\mu 1} & U_{\mu 2} & U_{\mu 3} \\
U_{\tau 1} & U_{\tau 2} & U_{\tau 3}
\end{array}\right) \\
& =\left(\begin{array}{ccc}
c_{12} c_{13} & s_{12} c_{13} & s_{13} e^{-\imath \delta} \\
-s_{12} c_{23}-c_{12} s_{13} s_{23} e^{\imath \delta} & c_{12} c_{23}-s_{12} s_{13} s_{23} e^{\imath \delta} & c_{13} s_{23} \\
s_{12} s_{23}-c_{12} s_{13} c_{23} e^{\imath \delta} & -c_{12} s_{23}-s_{12} s_{13} c_{23} e^{\imath \delta} & c_{13} c_{23}
\end{array}\right)
\end{aligned}
$$

where $c_{i j} \equiv \cos \theta_{i j}, s_{i j} \equiv \sin \theta_{i j}$ and $\delta$ is the Dirac CP violating phase which appears in neutrino oscillations. The matrix $P$ contains two Majorana phases unobservable in neutrino oscillation, but relevant to neutrinoless double beta decay [14]:

$$
P=\left(\begin{array}{ccc}
e^{\imath \alpha} & 0 & 0 \\
0 & e^{\imath \beta} & 0 \\
0 & 0 & 1
\end{array}\right)
$$

Combining Eq. (8) with the seesaw formula of Eq. (66) and solving for the right-handed neutrino mass matrix we find

$$
\begin{aligned}
M_{R} & =c^{2} M_{l} M_{\nu}^{-1} M_{l} \\
& =\frac{c^{2} m_{\tau}^{2}}{m_{1}}\left(\begin{array}{ccc}
\frac{m_{e}}{m_{\tau}} & 0 & 0 \\
0 & \frac{m_{\mu}}{m_{\tau}} & 0 \\
0 & 0 & 1
\end{array}\right) U_{P M N S} P^{2}\left(\begin{array}{ccc}
1 & 0 & 0 \\
0 & \frac{m_{1}}{m_{2}} & 0 \\
0 & 0 & \frac{m_{1}}{m_{3}}
\end{array}\right) U_{P M N S}^{T}\left(\begin{array}{ccc}
\frac{m_{e}}{m_{\tau}} & 0 & 0 \\
0 & \frac{m_{\mu}}{m_{\tau}} & 0 \\
0 & 0 & 1
\end{array}\right) .
\end{aligned}
$$

This enables us to establish a link between high scale parameters and low scale observables. 
We define a small expansion parameter $\epsilon$ as

$$
\epsilon=\frac{m_{\mu}}{m_{\tau}} \simeq 0.059
$$

in terms of which we have

$$
m_{e}=a_{e} \epsilon^{3} m_{\tau}, \frac{m_{1}}{m_{3}}=a_{13} \epsilon, \theta_{13}=t_{13} \epsilon, \theta_{23}=\frac{\pi}{4}+t_{23} \epsilon .
$$

Here $a_{e}, a_{13}, t_{13}$ and $t_{23}$ are $\lesssim \mathcal{O}(1)$ parameters with $a_{e}=1.400$. These expansions follow from low energy data assuming the picture of hierarchical neutrino masses.

We find that the requirement of generating adequate baryon asymmetry places significant constraints on the neutrino mixing parameters. Specifically, the following expansions

$$
\frac{m_{1}}{m_{2}}=\tan ^{2} \theta_{12}+a_{12} \epsilon \text { and } \beta=\alpha+\frac{\pi}{2}+b \epsilon,
$$

where $a_{12}$ and $b$ are $\lesssim \mathcal{O}(1)$ parameters are required. To see this, we note that the CP asymmetry parameter $\epsilon_{1}$ generated in the decay of $N_{1}$ is too small, of order $\varepsilon_{1} \sim \frac{\epsilon^{6}}{8 \pi} \sim 2 \times 10^{-9}$ if $a_{12}$ or $b$ are much greater than 1 . This is because the heavy neutrino masses would be strongly hierarchical in this case, $M_{1}: M_{2}: M_{3} \sim \epsilon^{6}: \epsilon^{2}: 1$. This can be altered to a weak hierarchy $M_{1}: M_{2}: M_{3} \sim \epsilon^{4}: \epsilon^{2}: 1$ by observing that the elements of the 2-3 block of $M_{R}$ of Eq. (11) are all proportional to $\left\{\frac{m_{1}}{m_{2}} e^{2 i \beta} \cos ^{2} \theta_{12}+e^{2 i \alpha} \sin ^{2} \theta_{12}\right\}$ and by demanding this quantity to be of order $\epsilon$. Eq. (13) is just this condition. $\varepsilon_{1} \sim \frac{\epsilon^{4}}{8 \pi} \sim 10^{-6}$ in this case, which can lead to acceptable baryon asymmetry, as we show.

An immediate consequence of Eq. (13) is that neutrinoless double beta decay is suppressed in the model. The effective mass relevant for this decay is found to be

$$
m_{\beta \beta}=\left|\sum_{i} U_{e i}^{2} m_{i}\right| \simeq\left|m_{2} e^{2 i \alpha} \epsilon\left(a_{12} c_{12}^{2}-2 i b s_{12}^{2}\right)+m_{3} s_{13}^{2} e^{-2 i \delta}\right|
$$

This is of the order $m_{3} \epsilon^{2} \sim 10^{-4} \mathrm{eV}$, which would be difficult to measure. This amplitude is small because of a cancelation between the leading contributions proportional to $m_{1}$ and $m_{2}$ (see Eq. (13) $)$.

In terms of these expansions, the r.h.n mass matrix becomes

$$
M_{R}=\left(\begin{array}{ccc}
A_{11} \epsilon^{5} & A_{12} \epsilon^{3} & A_{13} \epsilon^{2} \\
A_{12} \epsilon^{3} & A_{22} \epsilon^{2} & A_{23} \epsilon \\
A_{13} \epsilon^{2} & A_{23} \epsilon & A_{33}
\end{array}\right),
$$


where

$$
\begin{aligned}
& A_{11}=\frac{M_{\circ} \epsilon a_{e}^{2} e^{2 \imath \alpha} \cos 2 \theta_{12}}{\cos ^{2} \theta_{12}} \\
& A_{12}=-\frac{M_{\circ} \epsilon a_{e} e^{2 \imath \alpha} \tan \theta_{12}}{\sqrt{2}} \\
& A_{13}=\frac{M_{\circ} \epsilon a_{e} e^{2 \imath \alpha} \tan \theta_{12}}{\sqrt{2}} \\
& A_{22}=\frac{M_{\circ} \epsilon}{2}\left\{a_{13}-a_{12} e^{2 \imath \alpha} \cos ^{2} \theta_{12}-2 \imath b e^{2 \imath \alpha} \sin ^{2} \theta_{12}+2 e^{\imath(2 \alpha+\delta)} t_{13} \tan \theta_{12}\right\} \\
& A_{23}=\frac{M_{\circ} \epsilon}{2}\left\{a_{13}+a_{12} e^{2 \imath \alpha} \cos ^{2} \theta_{12}+2 \imath b e^{2 \imath \alpha} \sin ^{2} \theta_{12}\right\} \\
& A_{33}=-M_{\circ} \epsilon e^{2 \imath \alpha}\left\{t_{13} e^{\imath \delta} \tan \theta_{12}+\imath b \sin ^{2} \theta_{12}-\frac{a_{13} e^{-2 \imath \alpha}}{2}+\frac{a_{12} \cos ^{2} \theta_{12}}{2}\right\} .
\end{aligned}
$$

Here we defined $M_{\circ}=\frac{c^{2} m_{\tau}^{2}}{m_{1}}$. This hierarchical mass matrix is diagonalized by a series of rotations $U_{1}, U_{2}$ and $U_{3}$ such that;

$$
\left(K U_{3} U_{2} U_{1}\right) M_{R}\left(K U_{3} U_{2} U_{1}\right)^{T}=\left(\begin{array}{ccc}
\left|M_{1}\right| & 0 & 0 \\
0 & \left|M_{2}\right| & 0 \\
0 & 0 & \left|M_{3}\right|
\end{array}\right)
$$

where $K=\operatorname{diag}\left(k_{1}, k_{2}, k_{3}\right)$ with $k_{i}=e^{-\imath \phi_{i} / 2}$ being phase factors which make each r.h.n masses $M_{i}$ real, $M_{i}=\left|M_{i}\right| e^{\phi_{i}} . V=\left(K U_{3} U_{2} U_{1}\right)^{T}$ is the matrix that diagonalizes $M_{R}$. The unitary matrix $U_{1}$ is given by

$$
U_{1}=\left(\begin{array}{ccc}
1 & 0 & -\frac{A_{13}}{A_{33}} \epsilon^{2} \\
0 & 1 & 0 \\
\frac{A_{13}^{\star}}{A_{33}^{\star}} \epsilon^{2} & 0 & 1
\end{array}\right)
$$

Similarly, $U_{2}$ and $U_{3}$ are unitary matrices with off-diagonal entries given by

$$
\left(U_{2}\right)_{23}=-\frac{A_{23}}{A_{33}} \epsilon, \quad\left(U_{3}\right)_{12}=-\frac{\left(A_{12}-\frac{A_{13} A_{23}}{A_{33}}\right) \epsilon}{A_{22}-\frac{A_{23}^{2}}{A_{33}}} .
$$

The mass eigenvalues are found to be

$$
\begin{aligned}
M_{1} & =M_{\circ} k_{1}^{2} \epsilon^{5}\left(2 a_{13} a_{e}^{2} e^{2 \imath \alpha} \sin ^{2} \theta_{12}\right) \\
& \times\left(2 t_{13}^{2} e^{2 \imath(\alpha+\delta)} \sin ^{2} \theta_{12}+\left(a_{12}+2 \imath b+\left(a_{12}-2 \imath b\right) \cos 2 \theta_{12}\right) a_{13} \cos ^{2} \theta_{12}\right)^{-1}
\end{aligned}
$$




$$
\begin{aligned}
M_{2} & =M_{\circ} k_{2}^{2} \epsilon^{3} e^{2 \imath \alpha}\left(a_{13}\left(a_{12}+2 \imath b+\left(a_{12}-2 \imath b\right) \cos 2 \theta_{12}\right)+2 t_{13}^{2} e^{\imath(\delta+\alpha)} \tan ^{2} \theta_{12}\right) \\
& \times\left(-a_{13}+\imath b e^{2 \imath \alpha}+e^{2 \imath \alpha}\left(a_{12} \cos ^{2} \theta_{12}-\imath b \cos 2 \theta_{12}\right)+2 e^{\imath \delta} t_{13} \tan \theta_{12}\right)^{-1} \\
M_{3} & =\frac{M_{\circ} k_{3}^{2} \epsilon}{2}\left(a_{13}-\imath b e^{2 \imath \alpha}-e^{2 \imath \alpha}\left(a_{12} \cos ^{2} \theta_{12}-\imath b \cos 2 \theta_{12}+2 t_{13} e^{\imath \delta} \tan \theta_{12}\right)\right) .
\end{aligned}
$$

We use these results in the next section to determine $\varepsilon_{1}$.

\section{CP violation and lepton asymmetry}

Now that we have developed our framework, we can turn attention to the evaluation of the CP asymmetry $\varepsilon_{1}$ generated in the decay of the lightest r.h.n $N_{1}$. This arises from the interference between the tree-level and one-loop level decay amplitudes. ${ }^{6}$ In a basis where the r.h.n mass matrix is diagonal and real, the asymmetry in the decay of $N_{i}$ is given by [15]

$$
\varepsilon_{i}=-\frac{1}{8 \pi v^{2}\left(M_{D}^{\dagger} M_{D}\right)_{i i}} \sum_{j=2,3} \operatorname{Im}\left[\left(M_{D}^{\dagger} M_{D}\right)_{i j}\right]^{2}\left[f\left(\frac{M_{j}^{2}}{M_{i}^{2}}\right)+g\left(\frac{M_{j}^{2}}{M_{i}^{2}}\right)\right]
$$

where $f(x)$ and $g(x)$ represent the contributions from vertex and self energy corrections respectively. For the case of the non-supersymmetric standard model with right-handed neutrinos, these functions are given by [15]

$$
f_{\text {non-SUSY }}(x)=\sqrt{x}\left[-1+(x+1) \ln \left(1+\frac{1}{x}\right)\right], \quad g_{\text {non-SUSY }}(x)=\frac{\sqrt{x}}{x-1},
$$

while for the case of MSSM plus right-handed neutrinos, they are given by

$$
f_{\mathrm{SUSY}}(x)=\sqrt{x} \ln \left(1+\frac{1}{x}\right), \quad g_{\mathrm{SUSY}}(x)=\frac{2 \sqrt{x}}{x-1} .
$$

Here $v$ is the SM Higgs doublet VEV, $v \simeq 174 \mathrm{GeV}$. For the case of MSSM, $v$ in Eq. (21) is replaced by $v \sin \beta$. Hereafter, for definiteness in the numerical evaluation of the Boltzmann equations, we assume the SM scenario. However, our result should be approximately valid for the MSSM case as well. ${ }^{7}$ Assuming a mass hierarchy $M_{1} \ll M_{2}<M_{3}$ in the right-handed

\footnotetext{
${ }^{6}$ We will assume $M_{1} \ll M_{2}<M_{3}$. In this case, even if the heavier right-handed neutrinos $N_{2}$ and $N_{3}$ produce lepton asymmetry, it is usually erased before the decay of $N_{1}$.

${ }^{7}$ The function $f+g$ in MSSM is twice as big compared to the SM. However this is compensated by the factor $\frac{1}{g_{*}}$ that appears in $\eta_{B}$ which in MSSM is half of the SM value.
} 
neutrino sector i.e., $(x \gg 1)$, which is realized in our model, see Eq. (15), the above formula is simplified to the following one:

$$
\varepsilon_{1}=-\frac{3}{16 \pi v^{2}\left(M_{D}^{\dagger} M_{D}\right)_{11}} \sum_{k=2,3} \operatorname{Im}\left[\left(M_{D}^{\dagger} M_{D}\right)_{1 k}^{2}\right] \frac{M_{1}}{M_{k}} .
$$

$\varepsilon_{1}$ depends on the $(1,1),(1,2)$ and $(1,3)$ entries of $M_{D}^{\dagger} M_{D}$. These quantities can be related to the light neutrino mass and mixing parameters measurable in low energy experiments. In the basis where $M_{R}$ is diagonal, these elements are

$$
\begin{aligned}
& \left(M_{D}^{\dagger} M_{D}\right)_{11}=\left(c m_{\tau}\right)^{2}\left(V_{31} V_{31}^{*}+V_{21} V_{21}^{*} \epsilon^{2}+a_{e}^{2} V_{11} V_{11}^{*} \epsilon^{6}\right) \\
& \left(M_{D}^{\dagger} M_{D}\right)_{12}=\left(c m_{\tau}\right)^{2}\left(V_{31} V_{32}^{*}+V_{21} V_{22}^{*} \epsilon^{2}+a_{e}^{2} V_{11} V_{12}^{*} \epsilon^{6}\right) \\
& \left(M_{D}^{\dagger} M_{D}\right)_{13}=\left(c m_{\tau}\right)^{2}\left(V_{31} V_{33}^{*}+V_{21} V_{23}^{*} \epsilon^{2}+a_{e}^{2} V_{11} V_{13}^{*} \epsilon^{6}\right)
\end{aligned}
$$

where $V=K U_{3} U_{2} U_{1}$ is the unitary matrix diagonalizing $M_{R}$. Straightforward calculations give, to leading order in $\epsilon$,

$$
\begin{aligned}
\left(M_{D}^{\dagger} M_{D}\right)_{11}= & 8 a_{e}^{2} c^{2} m_{\tau}^{2} \epsilon^{4} \cos ^{2} \theta_{12} \sin ^{2} \theta_{12}\left(a_{13}^{2}+t_{13}^{2} \tan ^{2} \theta_{12}\right) \\
\times & 1 /\left\{8 t_{13}^{4} \sin ^{4} \theta_{12}+32 a_{13} t_{13}^{2} b \cos ^{2} \theta_{12} \sin ^{4} \theta_{12} \sin 2(\alpha+\delta)\right. \\
+ & a_{13} \cos ^{4} \theta_{12}\left[4 a_{13}\left(a_{12}^{2}-b^{2}\right) \cos 2 \theta_{12}+a_{13}\left(a_{12}^{2}+4 b^{2}\right)\left(3+\cos 4 \theta_{12}\right)\right. \\
+ & \left.\left.16 a_{12} t_{13}^{2} \sin ^{2} \theta_{12} \cos 2(\alpha+\delta)\right]\right\} \\
\left(M_{D}^{\dagger} M_{D}\right)_{12}^{2}= & 2 a_{e}^{2} c^{4} m_{\tau}^{4} \epsilon^{6} \tan ^{2} \theta_{12} e^{-\imath\left(\phi_{1}-\phi_{2}\right)} e^{-2 \imath(2 \alpha+\delta)}\left\{4\left(a_{13}^{2}-t_{13}^{2}\right) \cos 2 \theta_{12}-2 t_{13} \sin 2 \theta_{12}\right. \\
& \left.\left(2 a_{13} e^{\imath(2 \alpha+\delta)}-\left(a_{12}+2 \imath b\right) e^{-\imath \delta}\right)+4\left(a_{13}^{2}+t_{13}^{2}\right)+t_{13} \sin 4 \theta_{12} e^{-\imath \delta}\left(a_{12}-2 \imath b\right)\right\}^{2} \\
\times & 1 /\left\{\left[\imath b e^{\imath \delta}-a_{13} e^{-\imath(2 \alpha+\delta)}+a_{12} e^{-\imath \delta} \cos \theta_{12}-\imath b e^{-\imath \delta} \cos 2 \theta_{12}+2 t_{13} \tan \theta_{12}\right]^{2}\right. \\
\times & {\left[3 a_{12} a_{13}-2 \imath a_{13} b+4 t_{13}^{2} e^{-2 \imath(\alpha+\delta)}+4 \cos 2 \theta_{12}\left(a_{12} a_{13}-t_{13}^{2} e^{-2 \imath(\alpha+\delta)}\right)+\right.} \\
& \left.\left.a_{13}\left(a_{12}+2 \imath b\right) \cos 4 \theta_{12}\right]^{2}\right\} \\
& \\
= & 2 a_{e}^{2} c^{4} m_{\tau}^{4} \epsilon^{4} \sin ^{2} \theta_{12} e^{-\imath\left(\phi_{1}-\phi_{3}\right)}\left(a_{13} \cos \theta_{12}+e^{-\imath(2 \alpha+\delta)} t_{13} \sin \theta_{12}\right)^{2} \\
\times & 1 /\left\{a_{13} \cos ^{2} \theta_{12}\left(a_{12}-2 \imath b+\left(a_{12}+2 \imath b\right) \cos 2 \theta_{12}\right)+2 t_{13}^{2} \sin ^{2} \theta_{12} e^{-2 \imath(\alpha+\delta)}\right\}^{2}(28) \\
\left(M_{D}^{\dagger} M_{D}\right)_{13}^{2}(27) &
\end{aligned}
$$

These analytical expressions have been checked numerically. In Figure (1) we have plotted $\left|\varepsilon_{1}\right|$ as function of $\theta_{13}$ for fixed values of other observables. The solid line in Fig (1) 


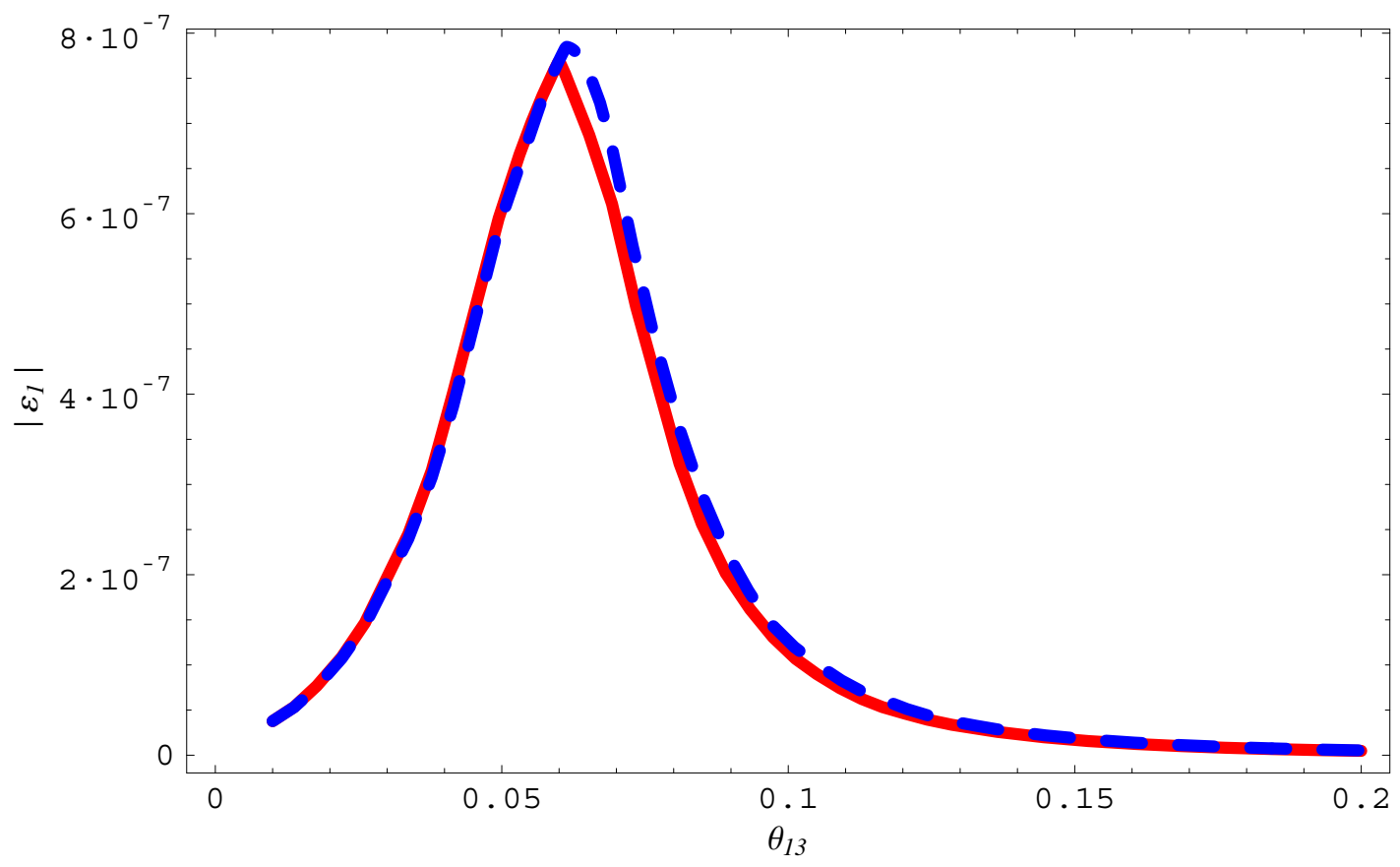

Figure 1: Plots for CP asymmetry parameter $\varepsilon_{1}$ using analytical (dotted) and numerical (solid) results as a function of the neutrino oscillation angle $\theta_{13}$. The input parameters used are $a_{12}=1, b=1, \Delta m_{\odot}^{2}=2.5 \times 10^{-5} \mathrm{eV}^{2}, \Delta m_{a}^{2}=5.54 \times 10^{-3} \mathrm{eV}^{2}$ and $\{\delta, \alpha\}=\{\pi / 4, \pi / 4\}$. Our model requires $\left|\varepsilon_{1}\right| \gtrsim 1.3 \times 10^{-7}$ to successfully generate an adequate number for the BA. This criterium happens to be satisfied only in the region for which $0.01 \lesssim \theta_{13} \lesssim 0.07$, this interval is not too sensitive to variations in the input parameters.

which corresponds to the exact numerical evaluation agrees very well with the dashed line corresponding to the analytical expressions.

From Figure (1), it is apparent that $\theta_{13}$ is constrained in the model from cosmology. If $\varepsilon_{1}<1.3 \times 10^{-7}$, the induced baryon asymmetry would be too small to explain observations. As can be seen from Figure (1), $\theta_{13}$ should lie in the range $0.01-0.07$ for an acceptable value of $\varepsilon_{1}$. This result does not change very much with variations in the other input parameters.

Electroweak sphaleron processes [6] will convert the induced lepton asymmetry to baryon asymmetry. The ratio of baryon asymmetry to entropy $Y_{B}$ is related to the lepton asymmetry 
through the relation [16]:

$$
Y_{B}=C Y_{B-L}=\frac{C}{C-1} Y_{L}
$$

where $C=\frac{8 N_{f}+4 N_{\varphi}}{22 N_{f}+13 N_{\varphi}}, N_{f}=3$ and $N_{\varphi}=1,2$ in the case of the SM and MSSM respectively. In either case $C \sim \frac{1}{3}$. In Eq. (29), $Y_{B}=\frac{n_{B}}{s}$ with $s=7.04 n_{\gamma}$.

There has been considerable interest in obtaining approximate analytical expression for baryon asymmetry [17, 18]. In order to estimate this, the dilution factor, often referred to as the efficiency factor $\kappa$ that takes into account the washout processes (inverse decays and lepton number violating scattering) has to be known. As an example, $\kappa=(2 \pm 1) \times$ $10^{-2}\left(\frac{0.01 \mathrm{eV}}{\widetilde{m}_{1}}\right)^{1.1 \pm 0.1}$ has been suggested in Ref. [17] from which $\eta_{B} \simeq 0.96 \times 10^{-2} \varepsilon_{N_{1}} \kappa$ has been calculated. In our work we solve the coupled Boltzmann equations numerically to estimate the baryon asymmetry without referring to the efficiency factor.

\section{Boltzmann equations}

In this section we set up the Boltzmann equations for computing the baryon asymmetry $\eta_{B}$ generated through the out of equilibrium decay of $N_{1}$. In our model the right-handed neutrino masses are not independent of the CP asymmetry parameter $\varepsilon_{1}$. Therefore a self consistent analysis within the model is required.

In the early universe, at temperature of order $N_{1}$ mass, the main thermal processes which enter in the production of the lepton asymmetry are the decay of the lightest r.h. neutrino, ${ }^{8}$ its inverse decay, and the lepton number violation scattering, $\Delta L=1$ Higgs exchange plus $\Delta L=2$ r.h.n exchange [19]. The production of the lepton asymmetry via the decay of the r.h.n is an out-of-equilibrium process which is most efficiently treated by means of the Boltzmann equations (BE).

The first BE which describes the evolution of the abundance of the r.h. neutrino and

\footnotetext{
${ }^{8}$ In our analysis we stick to the case where the asymmetry is due only to the decay of the lightest r.h. neutrino $N_{1}$.
} 
which corresponds to the source of the asymmetry is given by ${ }^{9}$

$$
\frac{d Y_{N_{1}}}{d z}=-\frac{z}{H s(z)}\left(\frac{Y_{N_{1}}}{Y_{N}^{e q}}-1\right)\left(\gamma_{D_{1}}+\gamma_{S_{1}}\right)
$$

where $z=\frac{M_{1}}{T}$. Here $s(z)$ is the entropy density and $\gamma_{D_{1}}, \gamma_{S_{1}}$ are the interaction rates for the decay and $\Delta L=1$ scattering contributions, respectively.

The second BE relevant to the lepton asymmetry is given by

$$
\frac{d Y_{B-L}}{d z}=-\frac{z}{s(z) H\left(M_{1}\right)}\left[\varepsilon_{1} \gamma_{D_{1}}\left(\frac{Y_{N_{1}}}{Y_{N}^{e q}}-1\right)+\gamma_{W} \frac{Y_{B-L}}{Y_{L}^{e q}}\right]
$$

where $\varepsilon_{1}$ is the $\mathrm{CP}$ violation parameter given by Eq. (21) and $\gamma_{W}$ is the washout factor which is responsible for damping of the produced asymmetry, see Eq. (49) below. In Eqs. (1301) and (31), $Y_{i}^{e q}$ is the equilibrium number density of a particle species $i$, which has a mass $m_{i}$, given by

$$
Y_{i}^{e q}(z)=\frac{45}{4 \pi^{4}} \frac{g_{i}}{g_{*}}\left(\frac{m_{i}}{M_{1}}\right)^{2} z^{2} K_{2}\left(\frac{m_{i} z}{M_{1}}\right)
$$

where $g_{i}$ is the particle internal degree of freedom $\left(g_{N_{i}}=2, g_{\ell}=4\right)$. At temperatures far above the electroweak scale one has $g_{*} \simeq 106.75$ in the standard model, and $g_{*} \simeq 228.75$ in MSSM. $H$, the Hubble parameter evaluated at $z=1$, and $s(z)$, the entropy density, are given by

$$
H=\sqrt{\frac{4 \pi^{3} g_{*}}{45}} \frac{M_{1}^{2}}{M_{P}} \quad, \quad s(z)=\frac{2 \pi^{2} g_{*}}{45} \frac{M_{1}^{3}}{z^{3}},
$$

where $M_{P}=1.22 \times 10^{19} \mathrm{GeV}$. We also have

$$
\gamma_{S_{j}}=2 \gamma_{t_{j}}^{(1)}+4 \gamma_{t_{j}}^{(2)}
$$

The decay reaction density $\gamma_{D_{j}}$ has the following expression:

$$
\gamma_{D_{j}}=n_{N_{j}}^{e q} \frac{K_{1}(z)}{K_{2}(z)} \Gamma_{N_{j}}
$$

where $K_{n}(z)$ are the modified Bessel functions. $\Gamma_{N_{j}}$ of the r.h.n $N_{j}$ is the tree level total decay rate defined as

$$
\Gamma_{N_{j}}=\frac{\left(\lambda^{\dagger} \lambda\right)_{j j}}{8 \pi} M_{j}
$$

\footnotetext{
${ }^{9}$ In this section we follow the notation of the first paper of Ref. 8 to which we refer the reader for further details.
} 
where

$$
n_{N_{i}}^{e q}(T)=\frac{g_{i} T m_{i}}{2 \pi^{2}} K_{2}\left(\frac{m_{i}}{T}\right) .
$$

We used the definition $\lambda=M_{D} / v$. We define the reaction density $\gamma^{(i)}$ of any process $a+b \rightarrow c+d$ by

$$
\gamma^{(i)}=\frac{M_{1}^{4}}{64 \pi^{4}} \frac{1}{z} \int_{\frac{\left(M_{a}+M_{b}\right)^{2}}{M_{1}^{2}}}^{\infty} d x \hat{\sigma}^{(i)}(x) \sqrt{x} K_{1}(\sqrt{x} z),
$$

where $\hat{\sigma}^{(j)}(x)$ are the reduced cross sections for the different processes which contribute to the Boltzmann equations. For the $\Delta L=1$ processes involving the quarks, we have

$$
\begin{aligned}
& \hat{\sigma}_{t_{j}}^{(1)}=3 \alpha_{u} \sum_{\alpha=1}^{3}\left(\lambda_{\alpha j}^{*} \lambda_{\alpha j}\right)\left(\frac{x-a_{j}}{x}\right)^{2}, \\
& \hat{\sigma}_{t_{j}}^{(2)}=3 \alpha_{u} \sum_{\alpha=1}^{3}\left(\lambda_{\alpha j}^{*} \lambda_{\alpha j}\right)\left(\frac{x-a_{j}}{x}\right)\left[\frac{x-2 a_{j}+2 a_{h}}{x-a_{j}+a_{h}}+\frac{a_{j}-2 a_{h}}{x-a_{j}} \ln \left(\frac{x-a_{j}+a_{h}}{a_{h}}\right)\right],
\end{aligned}
$$

where

$$
\alpha_{u}=\frac{\operatorname{Tr}\left(\lambda_{u}^{\dagger} \lambda_{u}\right)}{4 \pi} \simeq \frac{m_{t}^{2}}{4 \pi v^{2}}, \quad a_{j}=\left(\frac{M_{j}}{M_{1}}\right)^{2}, \quad a_{h}=\left(\frac{\mu}{M_{1}}\right)^{2},
$$

$\mu$ is the infrared cutoff which we set to $800 \mathrm{GeV}$ [19, 20]. For the $\Delta L=2$ r.h.n exchange processes, we have

$$
\begin{aligned}
& \hat{\sigma}_{N}^{(1)}=\sum_{\alpha=1}^{3} \sum_{j=1}^{3}\left(\lambda_{\alpha j}^{*} \lambda_{\alpha j}\right)\left(\lambda_{\alpha j}^{*} \lambda_{\alpha j}\right) A_{j j}^{(1)}+\sum_{\alpha=1}^{3} \sum_{n<j, j=1}^{3} \operatorname{Re}\left(\lambda_{\alpha n}^{*} \lambda_{\alpha j}\right)\left(\lambda_{\alpha n}^{*} \lambda_{\alpha j}\right) B_{n j}^{(1)} \\
& \hat{\sigma}_{N}^{(2)}=\sum_{\alpha=1}^{3} \sum_{j=1}^{3}\left(\lambda_{\alpha j}^{*} \lambda_{\alpha j}\right)\left(\lambda_{\alpha j}^{*} \lambda_{\alpha j}\right) A_{j j}^{(2)}+\sum_{\alpha=1}^{3} \sum_{n<j, j=1}^{3} \operatorname{Re}\left(\lambda_{\alpha n}^{*} \lambda_{\alpha j}\right)\left(\lambda_{\alpha n}^{*} \lambda_{\alpha j}\right) B_{n j}^{(2)}
\end{aligned}
$$

where

$$
\begin{aligned}
A_{j j}^{(1)} & =\frac{1}{2 \pi}\left[1+\frac{a_{j}}{D_{j}}+\frac{a_{j} x}{2 D_{j}^{2}}-\frac{a_{j}}{x}\left(1+\frac{x+a_{j}}{D_{j}}\right) \ln \left(\frac{x+a_{j}}{a_{j}}\right)\right] \\
A_{j j}^{(2)} & =\frac{1}{2 \pi}\left[\frac{x}{x+a_{j}}+\frac{a_{j}}{x+2 a_{j}} \ln \left(\frac{x+a_{j}}{a_{j}}\right)\right] \\
B_{n j}^{(1)} & =\frac{\sqrt{a_{n} a_{j}}}{2 \pi}\left[\frac{1}{D_{j}}+\frac{1}{D_{n}}+\frac{x}{D_{j} D_{n}}+\left(1+\frac{a_{j}}{x}\right)\left(\frac{2}{a_{n}-a_{j}}-\frac{1}{D_{n}}\right) \ln \left(\frac{x+a_{j}}{a_{j}}\right)\right. \\
& \left.+\left(1+\frac{a_{n}}{x}\right)\left(\frac{2}{a_{j}-a_{n}}-\frac{1}{D_{j}}\right) \ln \left(\frac{x+a_{n}}{a_{n}}\right)\right] \\
B_{n j}^{(2)} & =\frac{\sqrt{a_{n} a_{j}}}{2 \pi}\left\{\frac{1}{x+a_{n}+a_{j}} \ln \left[\frac{\left(x+a_{j}\right)\left(x+a_{n}\right)}{a_{j} a_{n}}\right]+\frac{2}{a_{n}-a_{j}} \ln \left(\frac{a_{n}\left(x+a_{j}\right)}{a_{j}\left(x+a_{n}\right)}\right)\right\}
\end{aligned}
$$


and

$$
D_{j}=\frac{\left(x-a_{j}\right)^{2}+a_{j} c_{j}}{x-a_{j}}, \quad c_{j}=a_{j} \sum_{\alpha=1}^{3} \frac{\left(\lambda_{\alpha j}^{*} \lambda_{\alpha j} \lambda_{\alpha j}^{*} \lambda_{\alpha j}\right)}{64 \pi^{2}} .
$$

Finally, $\gamma_{w}$ that accounts for the washout processes in the Boltzmann equations is

$$
\gamma_{W}=\sum_{j=1}^{3}\left(\frac{1}{2} \gamma_{D_{j}}+\frac{Y_{N_{j}}}{Y_{N_{j}}^{e q}} \gamma_{t_{j}}^{(1)}+2 \gamma_{t_{j}}^{(2)}-\frac{\gamma_{D_{j}}}{8}\right)+2 \gamma_{N}^{(1)}+2 \gamma_{N}^{(2)}
$$

Here, we emphasize the so-called RIS (real intermediate states) in the $\Delta L=2$ interactions which have to be carefully subtracted to avoid double counting in the Boltzmann equations.

This corresponds to the term $-\frac{1}{8} \gamma_{D_{j}}$ in Eq. (49). For more details see Refs. [17, 21] and the first paper of Ref. [18].

\section{Results and discussion}

We are now ready to present our numerical results. First we make several important remarks. Even though our model is supersymmetric, we have considered in our BE analysis only the SM particle interactions. This is a good approximation (see footnote 7 ). The authors in Ref. 20] have demonstrated that SUSY interactions do not significantly change the final baryon asymmetry. Furthermore, we have not included in our analysis the effects of renormalization group on the running masses and couplings. The first paper of Ref. [18] has studied these effects. This paper has also included finite temperature effects and $\Delta L=1$ scattering processes involving SM gauge bosons, which we have ignored in our analysis. This should be a good approximation since it is believed that these effects are significant in the weak washout regime and our model parameters seem to favor the strong washout regime with $\widetilde{m}_{1}=\frac{\left(M_{D}^{\dagger} M_{D}\right)_{11}}{M_{1}} \simeq 0.1 \mathrm{eV}$. Scattering processes involving gange bosons have also been studied in Ref. [21] in the context of resonant leptogenesis where they have been shown to be significant.

Our next step is to put this model to the test and check its predictions. In order to compute the value of the baryon asymmetry we proceed to numerically solve the Boltzmann equations. We scan the parameter space corresponding to the parameters $a_{12}, b$, the oscillation angle $\theta_{13}$, the CP phase $\delta$ and the Majorana phase $\alpha$. In order to automatically satisfy 
the oscillation data, we input the following light neutrino parameters:

$$
\Delta m_{\odot}^{2}=2.5 \times 10^{-5} \mathrm{eV}^{2}, \Delta m_{a}^{2}=5.54 \times 10^{-3} \mathrm{eV}^{2}, \sin \theta_{12}=0.52
$$

Using hierarchical spectrum, we see that the masses $m_{1}, m_{2}$ and $m_{3}$ are fixed. On the other hand we consider maximal mixing in the 2-3 sector of the leptonic mixing matrix, i.e $\theta_{23}=\frac{\pi}{4}+t_{23} \epsilon$ with $t_{23}$ being zero $\left(t_{23} \sim \mathcal{O}(1)\right.$ has minimal impact on $\left.\eta_{B}\right)$. The CP phase $\delta$ and the Majorana phase $\alpha$ are allowed to vary in the intervals $[0,2 \pi]$ and $[0, \pi]$ respectively. We remind the reader that the second Majorana phase $\beta$ is related to $\alpha$ through $\beta \simeq \alpha+\frac{\pi}{2}+b \epsilon$. $\theta_{13}$ will be allowed to vary in the interval $[0 ; 0.2]$ as it is bounded from above by reactor neutrino experiments.

In Figure (2), for a given set of input parameters, we illustrate the different thermally averaged reaction rates $\Gamma_{X}=\frac{\gamma_{X}}{n_{N_{1}}^{\text {eq }}}$ contributing to BE as a function of $z=\frac{M_{1}}{T}$.

All rates at $z=1$ fulfill the out of equilibrium condition (i.e. $\Gamma_{X} \lesssim H(z=1)$ ), and so the expected washout effect due to the $\Delta L=2$ processes will be small. The parameters chosen for this illustration are: $\delta=\pi / 2, \alpha=\pi / 2, a_{12}=0.01, b=0.9, c m_{\tau}=m_{t}\left(\frac{m_{\tau}}{m_{b}}\right)=135 \mathrm{GeV}$ and $\theta_{13}=0.02$. Eq. (50) fixes the light neutrino masses to be: $m_{1}=0.00271292 \mathrm{eV}$, $m_{2}=0.00688186 \mathrm{eV}$ and $m_{3}=0.0380442 \mathrm{eV}$. For this choice we obtain $\left|\epsilon_{1}\right| \simeq 2 \times 10^{-7}$. The calculated r.h.n masses in this case are

$$
M_{1}=9 \times 10^{9} \mathrm{GeV}, \quad M_{2}=8.7 \times 10^{11} \mathrm{GeV}, \quad M_{3}=2.6 \times 10^{14} \mathrm{GeV} .
$$

The mass of the lightest r.h.n is consistent with lower bound derived in Ref. [18, $M_{1} \geq$ $2.4 \times 10^{9} \mathrm{GeV}$, for hierarchical neutrino masses assuming that one starts with zero $N_{1}$ initial abundance (which is what we assumed in our calculation). This mass is also in accordance with the upper bound found in Ref. [22] following a model independent study of the CP asymmetry, and the bound derived in Ref. [17] based on the estimation of $\nu_{R}$ production and the study of the asymmetry washout.

Figure (3) represents the solution of the BE, $N_{1}$ abundance and the baryon asymmetry both as functions of $z$ for the same set of parameters mentioned above. The final baryon asymmetry, in terms of the baryon to photon ratio, is (see dark, solid curve in Fig. (3) for 
$z \gg 1)$

$$
\eta_{B} \simeq 6.03 \times 10^{-10} .
$$

This number is inside the observational range of Eq. (11). Our codes were tested to reproduce the results in the first paper of Ref. [8] before being applied to this model.

At this point we should mention some potential difficulties with gravitino decays in the model. If supersymmetry breaking is mediated by conventional supergravity, it is natural to expect the mass of the gravitino to be of order $1 \mathrm{TeV}$. Such a gravitino, with its Planck scale suppressed interactions, would decay into MSSM particles with a lifetime of order 1 second. The decay products would upset the success of standard big bang nucleosynthesis. In an inflationary scenario, demanding consistency with light element abundance puts a lower limit on the reheating temperature $T_{R}$. For gravitino mass in the range $300 \mathrm{GeV}$ to 3 $\mathrm{TeV}$, a limit $T_{R}>6 \times 10^{6} \mathrm{GeV}$ has been derived [23]. Now, the decays of $N_{1}$ that generates lepton asymmetry should occur after reheating, since any asymmetry produced prior to that will be diluted by inflation. Thus $M_{1}<T_{R}$ is required, which for gravitino mass in the range $300 \mathrm{GeV}$ to $3 \mathrm{TeV}$ is in conflict with the predictions of Eq. (51).

There are several ways around this problem. (i) In gauge mediated SUSY breaking scenario the gravitino is the lightest SUSY particle with mass in the range $10^{-4} \mathrm{eV}-100 \mathrm{GeV}$. For $m_{\tilde{g}}<100 \mathrm{MeV}$, there are no cosmological or astrophysical problems. In such a scenario the axion can serve as the dark matter. (ii) In anomaly mediated SUSY breaking scenario, the gravitino mass is enhanced by a loop factor compared to the squark masses and is naturally of order $100 \mathrm{TeV}$. Such a gravitino would decay with a shorter lifetime without affecting big bang nucleosynthesis. The gaugino is a natural dark matter candidate in this case. (iii) The gravitino itself can be the LSP and dark matter with a mass of order $100 \mathrm{GeV}$, in which case it does not decay [24]. Other solutions include changing the dynamics of the leptogenesis process by invoking (iii) non-thermal leptogenesis [25], (iv) resonant leptogenesis [21, 26], or (v) soft leptogenesis [27]. The results presented here are compatible with any one of the first three alternatives. 


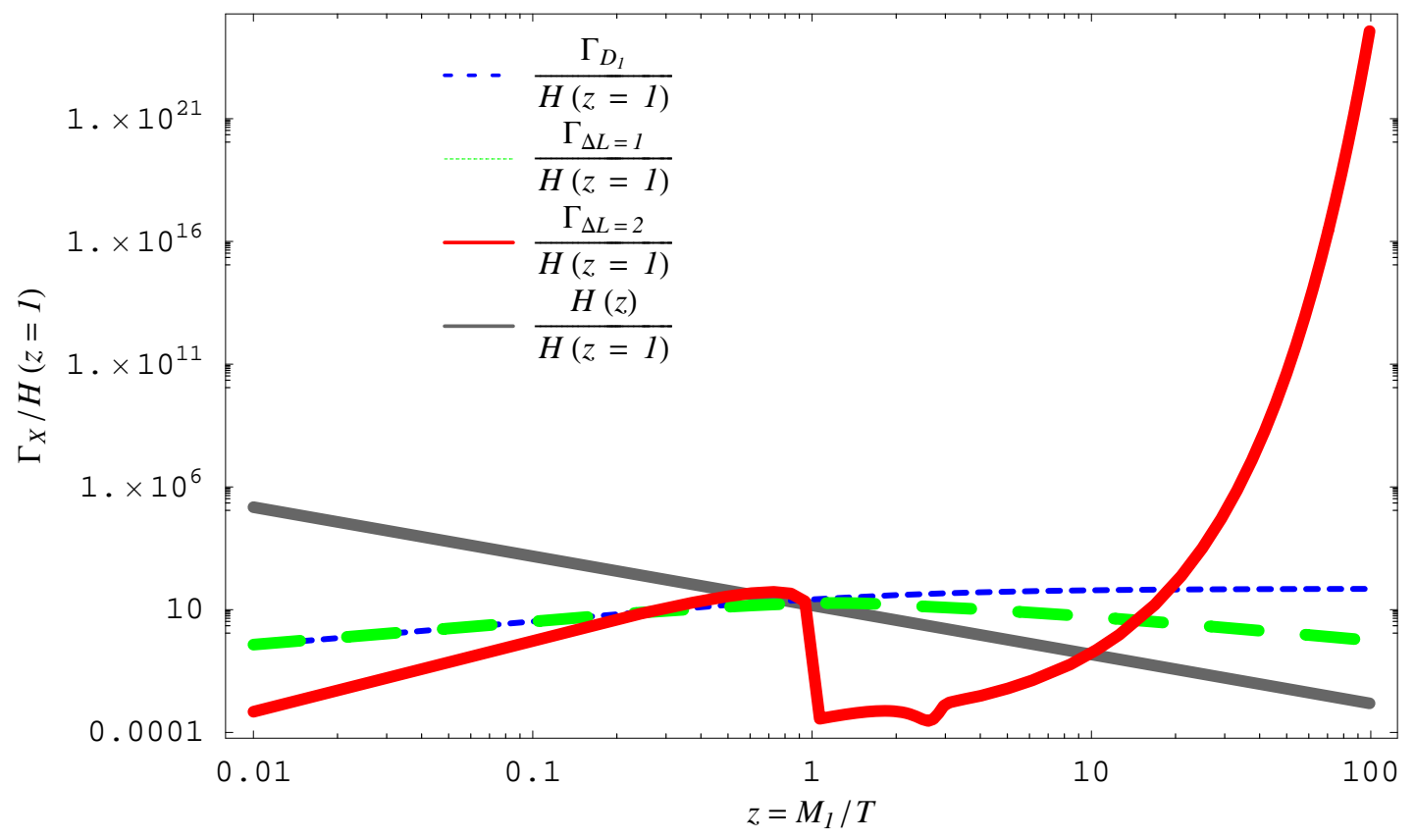

Figure 2: Various thermally averaged reaction rates $\Gamma_{X}$ contributing to BE normalized to the expansion rate of the Universe $H(z=1)$. The straight greyed line represents $H(z) / H(z=1)$, the dashed is for $\Gamma_{D_{1}} / H(z=1)$, the dotted-dashed line represents $\Gamma_{\Delta L=1} / H(z=1)$ processes and the red curve represents $\Gamma_{\Delta L=2} / H(z=1)$. 


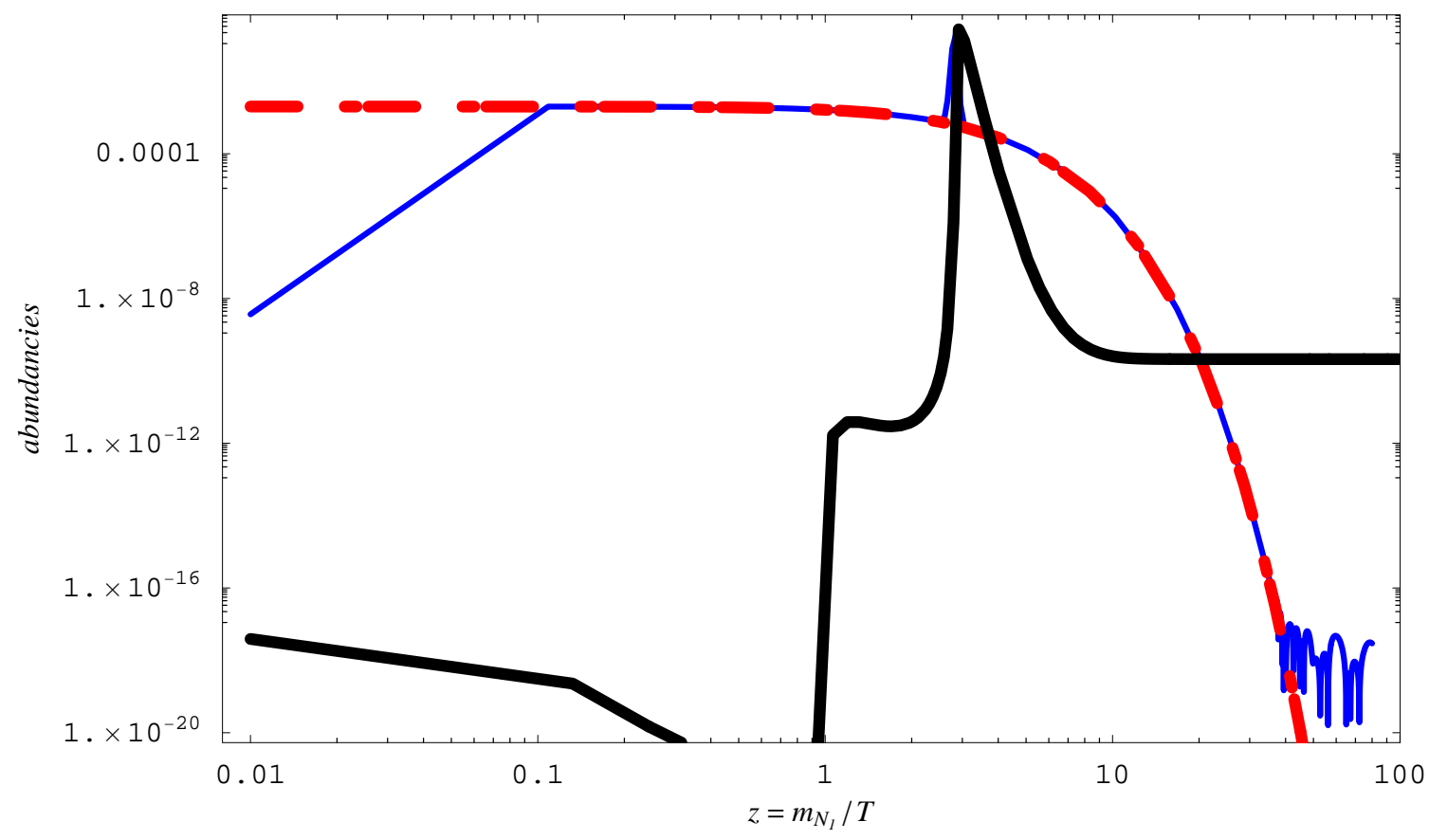

Figure 3: Evolution of $Y_{N_{1}}$ (solid blue), $Y_{N 1}^{e q}$ (dot-dash) and the baryon asymmetry $\eta_{B}$ (dark solid line) in terms of $\mathrm{z}$ in the model. The estimated value for the baryon asymmetry is $\eta_{B} \simeq 6.03 \times 10^{-10}$, with $Y_{N_{1}}^{i n i}=0$ and assuming no pre-existing $B-L$ asymmetry. 


\section{Conclusion}

An attractive feature of the seesaw mechanism is that it can explain the origin of small neutrino masses and at the same time account for the observed baryon asymmetry in the universe by the out of equilibrium decay of the super-heavy right handed neutrinos. It is then very tempting to seek a link between the baryon asymmetry parameter $\eta_{B}$ induced at high temperature and neutrino mass and mixing parameters observable in low energy experiments. No quantitative connection can be found between them in the SM. There have been several attempts in the literature [8, 9, 28, to establish a relationship between the two. In this paper we have addressed this question in the context of a class of minimal left-right symmetric models.

In the models under consideration the minimality of the Higgs sector implies that $M_{l}$ and $M_{D}$ (charged lepton and Dirac neutrino mass matrices) are proportional. As a result, the entire seesaw sector (including the heavy right-handed neutrinos and the light neutrinos) has only 9 parameters. This is the same number as low energy neutrino observables (3 masses, 3 mixing angles and 3 phases). As a result we are able to link the baryon asymmetry of the universe to low energy neutrino observables. This feature is unlike the SM seesaw which has too many arbitrary parameters. Our numerical solution to the coupled Boltzmann equations shows that this constrained system with $M_{l} \propto M_{D}$ leads to an acceptable baryon asymmetry. The requirement of an acceptable baryon asymmetry restricts some of the light neutrino observables. We find that $\tan ^{2} \theta_{12} \simeq m_{1} / m_{2}, 0.01 \lesssim \theta_{13} \lesssim 0.07$ and $\beta \simeq \alpha+\pi / 2$ are needed for successful baryogenesis. Future neutrino oscillation experiments can directly probe into the dynamics of the universe in its early stages.

\section{Acknowledgements}

One of the authors (A. B) wishes to thank I. Gogoladze for discussions and A. Strumia for useful correspondence. The work of K. B and A. B is supported in part by US Department of Energy grant \#DE-FG02-04ER46140 and \#DE-FG02-04ER41306. 


\section{References}

[1] S. Fukuda et al. [Super-Kamiokande Collaboration], Phys. Lett. B539, 179 (2002); Q. R. Ahmad et al. [SNO Collaboration], Phys. Rev. Lett. 89, 011302 (2002); B.T. Cleveland et al. Astrophys. J. 496, 505 (1998); D. N. Abdurashitov et al. (SAGE Collaboration), Phys. Rev. D60, 055801 (1999); W. Hampel et al. (GALLEX Collaboration), Phys. Let. B447, 127 (1999); C. Cattadori, (GNO Collaboration), Nucl. Phys. B111 (Proc. Suppl.), 311 (2002); M. Apollonio et al. [CHOOZ Collaboration], Phys. Lett. B466, 415 (1999); K. Eguchi et al. [KamLAND Collaboration], Phys. Rev. Lett. 90, 021802 (2003).

[2] P. Minkowski, Phys. Lett. B67, 421 (1977); T. Yanagida, proceedings of the Workshop on Unified Theories and Baryon Number in the Universe, Tsukuba, Japan 1979 (edited by A. Sawada and A. Sugamoto, KEK Report No. 79-18, Tsukuba); S.L. Glashow, in Quarks and Leptons, Cargése, eds. M. Lévy, et al., (Plenum, 1980, New York), p. 707; M. Gell-Mann, P. Ramond and R. Slansky, in Supergravity, ed. by P. van Nieuwenhuizen and D. Z. Freedman (North Holland, Amsterdam, 1979), p.315; R. N. Mohapatra and G. Senjanovic, Phys. Rev. Lett. 44, 912 (1980).

[3] M. Fukugita and T. Yanagida, Phys. Lett. B174, 45 (1986).

[4] D. N. Spergel et al., Astrophys. J. Suppl. 148, 175 (2003).

[5] A. D. Sakharov, JETP Letters 5, 24 (1967).

[6] V. A. Kuzmin, V. A. Rubakov and M. E. Shaposhnikov, Phys. Lett. B155, 36 (1985).

[7] K. S. Babu, B. Dutta and R. N. Mohapatra, Phys. Rev. D67, 076006 (2003); Phys. Rev. D60, 095004 (1999); Phys. Rev. D61, 091701 (2000); Phys. Rev. Lett. 85, 5064 (2000); Phys. Lett. B458, 93 (1999).

[8] G. C. Branco, R. Gonzalez Felipe, F. R. Joaquim, I. Masina, M. N. Rebelo and C. A. Savoy, Phys. Rev. D67, 073025 (2003); G. C. Branco, R. Gonzalez Felipe, F. R. Joaquim and M. N. Rebelo, Nucl. Phys. B640, 202 (2002). 
[9] W. Buchmüller and M. Plümacher, Phys. Lett. B389, 73 (1996); P. H. Frampton, S. L. Glashow and T. Yanagida, Phys. Lett. B548, 119 (2002); E. K. Akhmedov, M. Frigerio and A. Y. Smirnov, JHEP 0309, 021 (2003); S. Davidson and A. Ibarra, Nucl. Phys. B 648, 345 (2003); S. Pascoli, S. T. Petcov and W. Rodejohann, Phys. Rev. D68, 093007 (2003); J. C. Pati, Phys. Rev. D68, 072002 (2003); V. Barger, D. A. Dicus, H. J. He and T. j. Li, Phys. Lett. B583, 173 (2004); R. N. Mohapatra, S. Nasri and H. Yu, Phys. Lett. B615 231 (2005).

[10] G. Lazarides, Q. Shafi and C. Wetterich, Nucl. Phys. B181, 287 (1981); R. N. Mohapatra and G. Senjanovic, Phys. Rev. D23, 165 (1981); J. Schechter and J. W. F. Valle, Phys. Rev. D25, 774 (1982).

[11] T. Hambye and G. Senjanovic, Phys. Lett. B582, 73 (2004); S. Antusch and S. F. King, Phys. Lett. B597, 199 (2004).

[12] E. Ma, S. Sarkar and U. Sarkar, Phys. Lett. B458, 73 (1999); A. S. Joshipura, E. A. Paschos and W. Rodejohann, Nucl. Phys. B611, 227 (2001); W. Rodejohann and K. R. S. Balaji, Phys. Rev. D65, 093009 (2002); M. Frank, Phys. Rev. D70, 036004 (2004); U. A. Yajnik, J. Cline and M. Rabikumar, Pramana 62, 771 (2004); M. C. Chen and K. T. Mahanthappa, Phys. Rev. D71, 035001 (2005); N. Sahu and S. Uma Sankar, arXiv:hep-ph/0501069.

[13] Z. Maki, M. Nakagawa and S. Sakata, Prog. Theor. Phys. 28, 870 (1962); B. Pontecorvo, Sov. Phy. JETP 7, 172 (1958).

[14] S. M. Bilenky, J. Hosek and S. T. Petcov, Phys. Lett. B94, 495 (1980); J. Schechter and J. W. F. Valle, Phys. Rev. D22, 2227 (1980).

[15] M. Flanz, E. A. Paschos and U. Sarkar, Phys. Lett. B345, 248 (1995) [Erratum-ibid. B382, 447 (1996)]; L. Covi, E. Roulet and F. Vissani, Phys. Lett. B384, 169 (1996); M. Flanz, E. A. Paschos, U. Sarkar and J. Weiss, Phys. Lett. B389, 693 (1996); A. Pilaftsis, Phys. Rev. D56, 5431 (1997); W. Buchmüller and M. Plümacher, Phys. Lett. B431, 354 (1998). 
[16] S. Y. Khlebnikov and M. E. Shaposhnikov, Nucl. Phys. B308, 885 (1988). For a recent review see: W. Buchmuller, R. D. Peccei and T. Yanagida, arXiv:hep-ph/0502169.

[17] W. Buchmüller, P. Di Bari, M. Plümacher, Annals Phys. 315, 305 (2005).

[18] G. F. Giudice, A. Notari, M. Raidal, A. Riotto and A. Strumia, Nucl. Phys. B685, 89 (2004); R. Barbieri, P. Creminelli, A. Strumia and N. Tetradis, Nucl. Phys. B575, 61 (2000).

[19] M. A. Luty, Phy. Rev. D45, 455 (1992).

[20] M. Plümacher, Nucl. Phys. B530, 207 (1998); arXiv:hep-ph/9807557; K. Hamaguchi, arXiv:hep-ph/0212305.

[21] A. Pilaftsis and T. E. J. Underwood, Nucl. Phys. B692 303 (2004); arXiv:hep-ph/0506107.

[22] S. Davidson and A. Ibarra, Phys. Lett. B535, 25 (2002).

[23] K. Kohri, T. Moroi and A. Yotsuyanagi, arXiv:hep-ph/0507245.

[24] J. L. Feng, A. Rajaraman and F. Takayama, Phys. Rev. Lett. 91, 011302 (2003); J. L. Feng, S. Su and F. Takayama, Phys. Rev. D70, 075019 (2004).

[25] G. Lazarides and Q. Shafi, Phys. Lett. B258, 305 (1991); T. Asaka, K. Hamaguchi, M. Kawasaki and T. Yanagida, Phys. Rev. D61, 083512 (2000); R. Jeannerot and M. Postma, arXiv:hep-ph/0507162; S. Dar, Q. Shafi and A. Sil arXiv:hep-ph/0508037.

[26] A. Pilaftsis, Phys. Rev. D56, 5431 (1997); J. R. Ellis, M. Raidal and T. Yanagida, Phys. Lett. B546, 228 (2002).

[27] Y. Grossman, T. Kashti, Y. Nir and E. Roulet, Phys. Rev. Lett. 91, 251801 (2003); G. D’Ambrosio, G. F. Giudice and M. Raidal, Phys. Lett. B575, 75 (2003); L. Boubekeur, T. Hambye and G. Senjanovic, Phys. Rev. Lett. 93, 111601 (2004). 
[28] D. Falcone and F. Tramontano, Phys. Rev. D63, 073007 (2001); M. Hirsch and S. F. King, Phys. Rev. D64, 113005 (2001); T. Hambye, E. Ma and U. Sarkar, Nucl. Phys. B602, 23 (2001); M. S. Berger and K. Siyeon, Phys. Rev. D65, 053019 (2002); M. Fujii, K. Hamaguchi and T. Yanagida, Phys. Rev. D65, 115012 (2002); L. Boubekeur, arXiv:hep-ph/0208003 S. F. King, Phys. Rev. D67, 113010 (2003); L. Velasco-Sevilla, JHEP 0310, 035 (2003); T. Endoh, S. Kaneko, S. K. Kang, T. Morozumi and M. Tanimoto, J. Phys. G29, 1877 (2003); Y. Achiman; Phys. Lett. B599, 75 (2004); S. Dar, S. Huber, V. N. Senoguz and Q. Shafi, Phys. Rev. D69, 077701 (2004); W. l. Guo and Z. z. Xing, Phys. Lett. B583, 163 (2004); T. Hambye, J. MarchRussell and S. M. West, JHEP 0407, 070 (2004); C. H. Albright and S. M. Barr, Phys. Rev. D70, 033013 (2004); M. Raidal, A. Strumia and K. Turzynski, Phys. Lett. B609, 351 (2005); S. M. West, Phys. Rev. D71, 013004 (2005). 